\title{
Immunotherapy and gastroenteropancreatic neuroendocrine tumor
}

\author{
Timothy Allen*, Nepton Sheikh K, Shoja ER and Naveed Basha C \\ Global Allied Pharmaceuticals, Center for Excellence in Research and Development, 160 Vista Oak Dr. Longwood, FL 32779, USA
}

\begin{abstract}
Gastroenteropancreatic Neuroendocrine Tumor (GEP-NET) originates from the hormone producing cells of the gut along with rectum and pancreas. GEP-NET represents $75 \%$ of all neuroendocrine tumors (NETs). The incidence is 2.5 to 6.2 cases per 100,000 population and 5-year mean survival rate of $60 \%$ in the United States. No significant risk factor have been identified. But $25 \%$ of GEP-NETs have been associated with hereditary conditions like Multiple Endocrine Neoplasia type 1 (MEN-1), type-2 (MEN-2), Von Hippel Lindau disease (VHL gene), tuberous sclerosis complex (TSC) and neurofibromatosis type-1 (NF-1). Alterations and loss of various chromosomes result in the progression of GEP-NETs. Researchers are still challenged in exploring innate and adaptive immune systems. Immunotherapy has shown a promising development in the past few years. One optimistic treatment modality is immunotherapy, with much progress seen in the last decade. We review all the different classes of drugs, FDA approved or still under clinical trials directed at the therapy of the GEP-NET.
\end{abstract}

\begin{abstract}
Abbreviations: US: United States; FDA: US Food and Drug Administration; GEP-NET Gastroenteropancreatic Neuroendocrine Tumor; NET neuroendocrine tumors ; MEN-1; MEN-2 Multiple Endocrine Neoplasia type 1 ; type-2; VHL gene Von Hippel Lindau disease; TSC tuberous sclerosis complex; NF-1 neurofibromatosis type1 ; SDHD succinate dehydrogenase; FLT3 Fms-related tyrosine kinase 3; RAR- $\beta$ retinoic acid receptor- $\beta$; PDCD4 programmed cell death protein 4 ; $\mathrm{Rb}$ retinoblastoma RAR- $\beta$ tumor suppressor gene retinoic acid receptor- $\beta$; VEGFR2 vascular endothelial growth factor receptor 2 ; PDGFRb platelet-derived growth factor receptor b; FLT-3 FMSlike tyrosine kinase 3 ; TIE-2 TEK tyrosine kinase; TRKB endothelial; tropomyosin-related kinase B ; FKBP-12 FK Binding Protein-12; mTOR mammalian Target of Rapamycin; SSTR somatostatin receptors; TSGs: tumor suppressor genes; TP53: tumor protein p53; NTRK: neurotropic Tyrosine Kinase receptor gene; TK: tyrosine kinase; VEGF: vascular endothelial growth factor ; KDR: Kinase insert domain receptor; VEGF-2: VEGF KDR; HER: human epidermal growth factor receptor ; EML4: echinoderm microtubule-associated protein-like 4 gene; EGFR: epidermal growth factor receptor; KIF5B: kinesin family member $5 \mathrm{~B}$; RET: rearranged during transfection; LOH: Loss of heterozygosity; KRAS: Kirsten rat sarcoma viral oncogene homolog; SOX2: sex determining region Y-box2; BRAF: Raf murine sarcoma viral oncogene homolog B; AKT1: V-Akt Murine Thymoma Viral Oncogene Homolog; MAPK1: mitogen-activated protein kinase 1; MET: hepatocyte growth factor receptor; PI3K: phosphatidylinositol-4;5-bisphosphate 3-kinase; CA: catalytic unit alpha; TKI: Tyrosine Kinase inhibitors; RAS: Rat sarcoma gene; RAF: rapidly accelerated fibrosarcoma; MEK: Mitogenactivated protein kinases; ERK: extracellular signal-regulated kinases; SRC: sarcoma gene; DDR2: Discoidin domain-containing receptor 2; FGFR1: Fibroblast growth factor receptor 1; Rb: Retinoblastoma; MAB: Monoclonal Antibody; PD: Pharmacodynamics; PK: Pharmacokinetics; Ig: Immunoglobulin; ADCC: antibody-dependent cellular cytotoxicity; HGF: hepatocyte growth factor ; NeuGc-GM3: N-glycolylneuraminyllactosylceramide antibody; IGF-1R: insulin-like growth factor-1 receptor; NK: natural killer; ADC: antibody-drug conjugate; Vc: volume of distribution; TF: Tissue Factor; CEA: carcinoembryonic
\end{abstract}

antigen; RTK: receptor tyrosine kinase; ATP: Adenosine triphosphate; DNA: Deoxyribonucleic acid ; STAT: signal transducer and activator of transcription; KIT: mast/stem cell growth factor; FLT-3: FMS-like tyrosine kinase 3; TIE-2: TEK tyrosine kinase; endothelial; TRKB: tropomyosin-related kinase B; AXL: Tyrosine-protein kinase receptor UFO; MAPK or MEK: mitogen-activated protein kinase; CDC2: cyclin-dependent kinase 1; TEC: Tec protein tyrosine kinase; $\mathrm{PIP}_{3}$ : phosphatidylinositol-3,4,5-trisphosphate; BCR: B-cell antigen receptor; ATR: ataxia telangiectasia and rad3-related; Chk1: checkpoint kinase 1; CSF1R: colony-stimulating factor-1 receptor; wt: wild-type; EMT: epithelial-mesenchymal transition; TYRO3: TYRO3 protein tyrosine kinase; MER: Proto-oncogene tyrosine-protein kinase of MERTK gene; DCs: Dendritic cells; IFN: interferon; ROS1: C-ros oncogene 1 Ig: Immunoglobulin; APCs: antigen presenting cells; CTLs: cytotoxic T-lymphocytes; CTLA4: cytotoxic T-lymphocyte-associated antigen-4; Fc: fragment constant; CDC: complement-dependent cytotoxicity; TAAs: tumor-associated antigens; MHC: major histocompatibility complex; IgSF: immunoglobulin superfamily; HLA: Human Leukocyte Antigen; MVA: modified vaccinia virus Ankara; MUC1: mucin 1; IL: interleukin; CMV: cytomegalovirus; TLR: toll-like receptor; URLC10: up-regulated lung cancer 10; CDCA1: cell division cycle associated 1; KIF20A: kinesin-like family member 20A; MAGE-A3: melanoma antigen family A3; CIK: Cytokine-induced killer; CD: cluster of differentiation; Hsp: Heat Shock Protein

${ }^{\star}$ Correspondence to: Timothy Allen, Global Allied Pharmaceuticals, Center for Excellence in Research and Development, 160 Vista Oak Dr. Longwood, FL 32779, USA, E-mail: timallenmed69@gmail.com

Key words: gastroenteropancreatic neuroendocrine tumor (gep-net, neuroendocrine tumors (nets), multiple endocrine neoplasia type 1 (men-1), type-2 (men-2), von hippel lindau disease (vhl gene), tuberous sclerosis complex (tsc) and neurofibromatosis type-1 (nf-1), fda: us food and drug administration

Received: January 02, 2019; Accepted: January 14, 2019; Published: January 16, 2019 


\section{Introduction}

Gastroenteropancreatic Neuroendocrine Tumor (GEP-NET) originates from the hormone producing cells of the gut along with rectum and pancreas. In 2004, approximately 5.25 new cases per $1,00,000$ population were diagnosed for GEP-NET as compared to earlier 3 cases per 100,000 population [1]. GEP-NET represents $75 \%$ of all neuroendocrine tumors (NETs) [2]. According to the Surveillance, Epidemiology and End Results (SEER) Program database, the incidence is 2.5 to 6.2 cases per 100,000 population and 5-year mean survival rate of $60 \%$ in the United States [3]. There is an increase of about $400 \%$ in the incidence since the past 3 decades [4,5]. It includes different types of histopathological and genetic characteristics. The male-to-female ratio is 5.35:4.76 with the predominance of male over female and higher incidence observed between the age of 40 to 50 years [1].

GEP-NETs develop in hormone producing cells in the gut and pancreatic areas. They are classified on the basis of embryonic site of origin and the hormones secreted by them, in the course. The classifications of GEP-NETs are as follows:

1. Functional Carcinoids: Aberrant/excessive secretion of serotonin

2. Insulinoma: Aberrant/excessive secretion of insulin, pro-insulin and C-peptide

3. Gastrinoma: Aberrant/excessive secretion of gastrin

4. Glucagonoma: Aberrant/excessive secretion of glucagon

5. Somatostatinoma: Aberrant/excessive secretion of somatostatin

6. Vasoactive Intestinal Peptidoma: Aberrant/excessive secretion of vasoactive intestinal peptide (VIP)

The symptoms associated with GEP-NETs are dependent on the type of hormone secreted and include: carcinoid syndrome, ZollingerEllison syndrome, diabetes, hypoglycemia, gall stones, cutaneous and visceral ulcers, diarrhea and acromegaly [6].

\section{Etiology/Predisposing factors}

For a large proportion of GEP-NETs, no significant risk factor have been evident [7]. Some conditions like ulcerative colitis, preexisting diabetes mellitus and hypergastrinemia have been linked to this cancer [7-10]. However, the clear origin is still not known. Various risk factors associated with GEP-NET, include hereditary conditions like Multiple Endocrine Neoplasia type 1 (MEN-1), type-2 (MEN-2), Von Hippel Lindau disease (VHL gene), tuberous sclerosis complex (TSC) and neurofibromatosis type-1 (NF-1) [11-16]. Approximately, $25 \%$ of GEP-NETs have been associated with these risk factors. In such patients, NETs develop 15 years earlier than the stipulated age of the development of sporadic tumors [13,17]. In about $25 \%-75 \%$ of the patients with MEN-1, pancreatic NETs are proposed to develop [13].

In patients with Von-Hippel-Lindau disease, NETs are of benign origin $[13,15,18]$. NET is rarely found (1\%) in patients with NF-1 and TSC and hence, does not form the part of the major clinical feature. However, if present, it is observed in approximately $30 \%$ of the cases of somatostatinomas in patients with NF-1 [16].

\section{Pathophysiology and molecular basis [19]}

GEP-NETs are the tumors of embryonic origin. The mutations and loss of heterozygosity $(\mathrm{LOH})$ of several genes at different chromosomal locations forms the molecular basis of GEP-NETs.
Chromosome 11q: Unlike foregut NETs, the NET of midgut region does not exhibit MEN1 gene mutations. However, as per some studies, the NET of ileal and duodenal region does show the loss of $11 \mathrm{q}$ chromosome in $22 \%$ of cases. This region encodes for succinate dehydrogenase (SDHD), a tumor suppressor gene. These tumors also represent the loss of heterozygosity at the location of the SDHD gene present on the 11q23 chromosome [20]. Based on various studies, the NET of midgut origin also shows the loss of chromosomes at 9p location by $21 \%, 18$ p by $38 \%$ and $18 \mathrm{q}$ by $33 \%[21,22]$. The chromosomal gain at $17 \mathrm{q}$ and $19 \mathrm{p}$ site is reported in $57 \%$ of cases. Loss of heterozygosity is seen in $88 \%$ cases at chromosome 18 [23]. All these reports indicate that NET tumors follow alterations at various chromosomes and modification of different molecular pathways.

Chromosome 3p: Chromosome 3p, which is deleted in $30 \%$ of pancreatic NETs is associated with a number of genes which includes VHL, retinoic acid receptor- $\beta$ (RAR- $\beta$ ), MLH1 and RASSF1A. All of them belong to the class of tumor suppressor genes. RAR- $\beta$ is associated with the process of apoptosis and development of embryo. In a study conducted by Chan et al., it was found to be hypermethylated by $25 \%$ in gastric NETs and none in the pancreatic NETs [24]. In another study conducted by House et al., it was observed to be hypermethylated by $25 \%$ in pancreatic NETs [25]. A study conducted by House et al., for hMLH1 hypermethylation, which is a repairing gene for mismatching of DNA,hyper-methylation was found in $23 \%$ of pancreatic NETs [26]. RASSF1A is known to interact with Ras oncogene. It is present at $3 \mathrm{p} 21.3$ chromosomal loci. It is inactivated due to epigenetic hypermethylation caused by a promoter [27]. It also inhibits the concentration of cyclin D1, resulting in cell cycle arrest [28]. Hyper-methylation of RASSF1 A was observed in $32 \%$ of NETs and $83 \%$ in pancreatic NETs [29, 30].

Chromosome 11q13: In the vicinity of MEN1 gene, there exists Phospholipase C $\beta 3$ (PLCB3) on chromosome 11q13. It regulates the signal transduction via receptors. It also helps the programmed cell death protein 4 (PDCD4) genes to be expressed, which is a tumor suppressor gene. In a study conducted by Stalberg et al., in 82 cases of NETs, 18\% exhibited loss of expression [31].

Cell Cycle Regulators: Alterations in the cell cycle pathway are manifested in almost all the cancer [32]. Cyclin- dependent kinases-2,4 and $6(\mathrm{cdk} 2, \mathrm{cdk} 4 / 6)$ control the transition of cell cycle from one phase to another. Cyclin D1 leads to the phosphorylation and inactivation of retinoblastoma tumor suppressor gene $(\mathrm{Rb})$ and acts as an essential cofactor for $c \mathrm{dk} 4 / 6$. The inactivated $\mathrm{Rb}$ releases and advances E2F, a transcription factor, through restriction point. E2F regulates the metabolism of DNA along with cyclin E and A. Cyclin E phosphorylates the $\mathrm{Rb}$ completely by forming cyclinE-cdk2 complex.

The Cip/Kip family acts as an inhibitor of cdk2. It includes: $\mathrm{p} 21^{\mathrm{CIP} 1}$, p27 ${ }^{\mathrm{KIP1}}$ and $\mathrm{p} 57^{\mathrm{KIP} 2}$ [33]. INK4 proteins inhibit cdk4 and cdk6. INK4 proteins i.e- INK4a, INK4b, INK4c and INK4d, involve Rb and p53 tumor suppressor pathways and hence, restricts the tumor development. p $16^{\mathrm{INK} 4 \mathrm{a}}$ and $\mathrm{p} 14^{\mathrm{ARF}}$ are encoded by INK4a [34]. p16 $6^{\mathrm{INK} 4 \mathrm{a}}$ acts as a sequestering agent and forms a cdk4-cdk6 complex. This complex leads to the release of $\mathrm{p} 27^{\mathrm{KIP} 1}$, which acts as cyclinE/cdk2 inhibitor.

p53 stabilization and consequent cell cycle arrest are carried out by p14 ${ }^{\mathrm{ARF}}$. It inhibits MDM2, a negative regulator of p53. Another, cdk4/6 inhibitor, $\mathrm{p} 18^{\mathrm{INK} 4 \mathrm{C}}$ is encoded by INK4c gene [35].

Homozygous deletions or hyper-methylation of $\mathrm{p} 16^{\mathrm{INK} 4 \alpha} / \mathrm{p} 14^{\mathrm{ARF}}$ genes located at $9 \mathrm{p} 21$ chromosomal location have been associated with up to $92 \%$ of NETs in some studies. In a study conducted by Chan et al., hyper-methylation of $\mathrm{p} 16^{\mathrm{INK} 4 \mathrm{a}}$ and $\mathrm{p} 14^{\mathrm{ARF}}$ was exhibited in $31 \%$ and 
$44 \%$ GI NETs, while only $9 \%$ of hyper-methylation was identified in pancreatic NETs [24].

$\mathrm{p} 18^{\mathrm{INK} 4 \mathrm{c}} / \mathrm{p}^{27 \mathrm{KIP} 1}$ are also proposed to be involved in NET pathogenesis. In pancreatic NETs, the p18 location on 1 p32 chromosome is deleted, while p27 and p21 locus are not. According to a study conducted by Kawahara et al, the locus p21, present on chromosome 6 p12 was over expressed in $66 \%$ cases of GI NETs of malignant origin.

In a study, GI NETs of malignant and benign nature elucidated cyclin D1 oncogene (CCND1) over expression in 100\% and $94 \%$ cases, respectively [36]. CCND1 is located on 11q13 site of chromosome [37]. According to a study, $65 \%$ of pancreatic NETs were proposed to over express cyclin D1 [38].

\section{Immunotherapy}

Figure 1 represents various molecular pathways based immunotherapy (Figure 1).

\section{Kinase inhibitors}

FDA-Approved Kinase Inhibitors: Sunitinib is the only FDA approved immunotherapeutic for the treatment of pancreatic NETs.

Sunitinb: The orally bioavailable indolinone-based tyrosine kinase inhibitor with potential antineoplastic activity [39]. Sunitinib blocks the tyrosine kinase activities of vascular endothelial growth factor receptor 2 (VEGFR2), platelet-derived growth factor receptor b (PDGFRb), and c-kit, thereby inhibiting angiogenesis and cell proliferation. This agent also inhibits the phosphorylation of Fms-related tyrosine kinase 3 (FLT3), another receptor tyrosine kinase expressed by some leukemic cells.

Indication and use: Sunitinb is a kinase inhibitor indicated for the treatment of pNET in patients with unresectable locally advanced or metastatic disease.

Pharmacokinetics: $\mathrm{T}_{\max }: 6$ to 12 hours, $\mathrm{t}^{1 / 2}: 40-60$ hours. AUC and $\mathrm{C}_{\max }$ increases as the dose increases in the range of $25-100 \mathrm{mg}$.

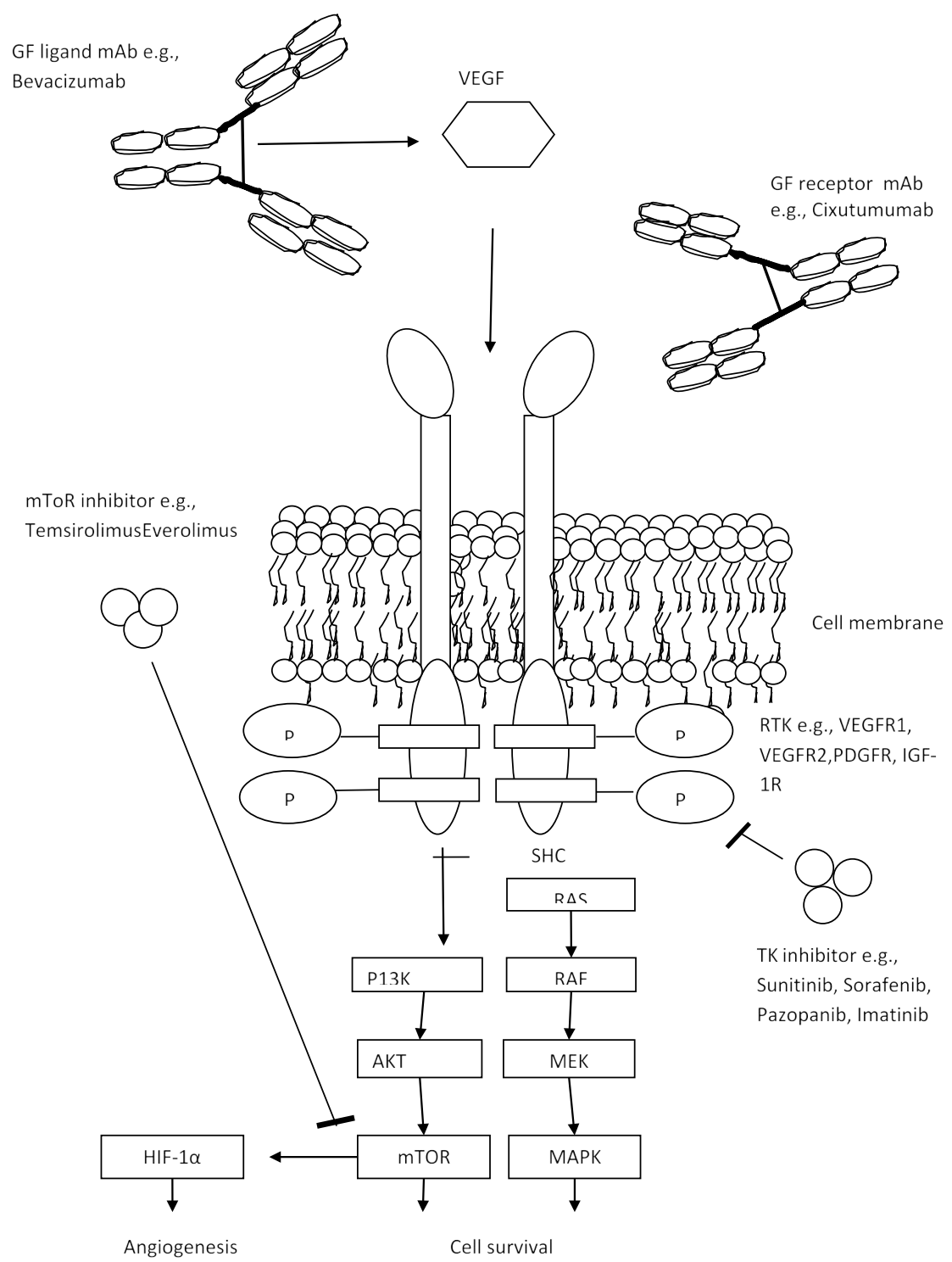

Figure 1. Targeted therapy for GEP-NETs [66] 
Warnings: Severe hepatotoxicity and deaths have been reported, hazards to pregnant women and fetus, prolonged QT interval, cardiac toxicity, hypertension, thyroid dysfunction, adrenal hemorrhage. It is advised that people undergoing surgery should stop taking sunitinib.

Adverse events: Various adverse reactions that are associated with sunitinib are weakness, fever, stomatitis, edema, rashes, discoloration of skin, cough, bleeding, pain in back, taste alteration, constipation, change in colour of hairs and texture of skin.

\section{Non-FDA approved Kinase Inhibitors}

Some of the kinase inhibitors that are under clinical trials in phase I-III are as mentioned in the Table 1.

\section{mTOR inhibitors}

\section{FDA-Approved mTOR Inhibitors}

Everolimus: A derivative of the natural macrocyclic lactone sirolimus with immunosuppressant and anti-angiogenic properties [56]. In cells, everolimus binds to the immunophilin FK Binding Protein-12 (FKBP-12) to generate an immunosuppressive complex that binds to and inhibits the activation of the mammalian Target of Rapamycin (mTOR), a key regulatory kinase. Inhibition of mTOR activation results in the inhibition of $\mathrm{T}$ lymphocyte activation and proliferation associated with antigen and cytokine (IL-2, IL-4, and IL15) stimulation and the inhibition of antibody production.

Indications \& use: Everolimus is a mTOR inhibitor that is indicated for adults with PNET that are unresectable, locally advanced or metastatic.

PD/PK: $\mathrm{T}_{\max }: 1-2$ hours, Elimination $\mathrm{t}^{1 / 2}: 30$ hours. With the increase in the dose within the range of $5 \mathrm{mg}$ to $70 \mathrm{mg}, \mathrm{C}_{\max }$ increases.

Warnings: Non-infectious pneumonitis, oral ulcers, renal failure, impaired wound healing, alterations in laboratory tests, vaccinations, embryo-fetal toxicity.

Adverse Events: The adverse reactions include reduced appetite, rashes, diarrhea, stomatitis, headache, and nausea, pain in abdomen, edema, cough, skin rashes and infection.

Non-FDA approved mTOR inhibitors: The below mentioned mTOR inhibitors are under clinical trials in Phase-II [57]:

\section{Temsirolimus}

An ester analog of rapamycin. Temsirolimus binds to and inhibits the mammalian target of rapamycin (mTOR), resulting in decreased expression of mRNAs necessary for cell cycle progression and arresting cells in the G1 phase of the cell cycle. mTOR is a serine/ threonine kinase which plays a role in the PI3K/AKT pathway that is upregulated in some tumors.

\section{Proteasome inhibitors}

Non-FDA approved proteasome inhibitors: There is no proteasome inhibitor that is currently approved by FDA for GEP-NETs. However, the below mentioned proteasome inhibitors are under clinical trials in Phase-II [58-59].

Carfilzomib: An epoxomicin derivate with potential antineoplastic activity. Carfilzomib irreversibly binds to and inhibits the chymotrypsinlike activity of the $20 \mathrm{~S}$ proteasome, an enzyme responsible for degrading a large variety of cellular proteins. Inhibition of proteasomemediated proteolysis results in an accumulation of polyubiquinated proteins, which may lead to cell cycle arrest, induction of apoptosis, and inhibition of tumor growth.

Bortezomib: It is $26 \mathrm{~S}$ proteasome inhibitor with anticancer properties. By inhibiting it, bortezomib hampers several signaling pathways. This results in cell cycle arrest, differentiation and antiangiogenesis. It also acts as NF-kappaB inhibitor and hence reduces cell survival, angiogenesis and growth of tumor.

\section{Monoclonal antibodies (MABs)}

Non-FDA Approved MABs: There are no MABs that are currently approved by FDA for GEP-NETs [60-65]. However, many MABs are under clinical trials in phase I-III as mentioned below:

Bevacizumab: It is a recombinant monoclonal antibody that acts against the pro-angiogenic cytokine, VEGF. It acts by adhering to VEGF and prevents it binding to its receptor, hence affecting tumor growth and associated blood vessels.

Ganitumab: It is an IGF-1R inhibitor monoclonal antibody having anti-neoplastic activity. It adheres to IGF-1R and triggers a cascade of signals resulting in inhibition of P13K/Akt pathway, thus, inhibiting cell proliferation and apoptosis.

Pertuzumab: It is an HER-2 tyrosine kinase receptor inhibitor monoclonal antibody. It inhibits dimerization of receptor and protein.

Table 1. Non-FDA approved kinase inhibitors [40-55]

\begin{tabular}{|c|c|c|c|c|}
\hline Kinase Inhibitors & Clinical trial identifier number & Phase & Study design & Target \\
\hline abozantinib & NCT01466036 & Phase-II & Safety/ Efficacy Study, Open Label & MET, VEGFR \\
\hline azopanib & NCT01841736; NCT01465659 & Phase-II; Phase-I/II & Efficacy Study, Double Blind; Safety/ Efficacy Study, Open Label & VEGFR, PDGFR \\
\hline Sorafenib & NCT00131911; NCT00942682 & Phase-II; Phase-I & Efficacy Study, Open Label; Safety Study, Open Label & TK Inhibitors \\
\hline Trebananib & NCT01548482 & Phase-I & Safety Study, Open Label & TK Inhibitors \\
\hline Motesanib & NCT00427349 & Phase-II & Treatment, Open Label & TK Inhibitors \\
\hline Vatalanib & NCT00227773 & Phase-II & Treatment & TK Inhibitors \\
\hline Axitinib & NCT01435122 & Phase-II & Safety/ Efficacy Study, Open Label & TK Inhibitors \\
\hline Regorafenib & NCT02259725 & Phase-II & Efficacy Study, Open Label & TK Inhibitors \\
\hline Gefitinib & NCT00075439 & Phase-II & Efficacy Study, Open Label & TK Inhibitors \\
\hline Dovitinib & NCT02108782 & Phase-II & Efficacy Study, Open Label & TK Inhibitor \\
\hline Famitinib & NCT01994213 & Phase-II & Safety/ Efficacy Study, Open Label & TK Inhibitors \\
\hline $\mathrm{X}-82$ & NCT01784861 & Phase-I/II & Safety/ Efficacy Study, Open Label & TK Inhibitor \\
\hline Dactolisib (BEZ235) & NCT01628913 & Phase-II & $\begin{array}{c}\text { Safety/ } \\
\text { Efficacy Study, Open Label }\end{array}$ & PI3K/mTOR Inhibitor \\
\hline BYL-719 & NCT02077933 & Phase-I & Safety/ Efficacy Study, Open Label & PI3K Inhibitor \\
\hline
\end{tabular}


This results in inactivation of HER signaling pathways and favors apoptosis.

Cixutumumab: It is a complete human monoclonal body derived from IgG1 and possesses anti-neoplastic activity. It adheres to the IGF$1 \mathrm{R}$ and hence, inhibits the PI3K/AKT pathway activation resulting in tumor cell death.

\section{Heat shock protein inhibitors:}

Non-FDA Approved Heat Shock Protein Inhibitors: There are no heat shock protein inhibitors that are currently approved by FDA for GEP-NETs. However, many are under clinical trials in phase I-III as mentioned in the Table 2.

\section{Cytokine therapy}

Non-FDA approved cytokine therapy: There is no cytokine therapy that is currently approved by FDA for GEP-NETs. However, the cytokine therapy available for GEP-NETs that are under clinical trials in phase I-III are as in the Table 3:

\section{HDAC inhibitor}

Non-FDA approved hdac inhibitor: There is no HDAC inhibitor that is currently approved by FDA for GEP-NETs [71]. However, some therapies are under clinical trials in phase I-III for GEP-NETs as mentioned below:

Romidepsin: It is an antineoplastic antibiotic derived from the bacterium Chromobacterium violaceum. It is an HDAC inhibitor that alters gene expression, resulting in the halt of cell cycle, apoptosis and inhibition of proliferation of cells.

\section{Growth factor inhibitors}

Non-FDA approved growth factor inhibitors: There is no growth factor inhibitor that is currently approved by FDA for GEP-NETs [7273]. Clinical trials undergoing in phase I-III are as mentioned below:

Ziv-Aflibercept: It is a recombinant protein that blocks VEGF to adhere to its respective receptor resulting in inhibition of angiogenesis, metastasis and tumor growth.

\section{Somatostatin analogues}

\section{FDA approved somatostatin analogues}

Lanreotide: It was approved by the FDA On December 16, 2014, the U. S. Food and Drug Administration approved lanreotide (Somatuline Depot Injection, Ipsen Pharma) for the treatment of patients with unresectable, well or moderately differentiated, locally advanced or metastatic gastroenteropancreatic neuroendocrine tumors (GEP-NETs) to improve progression-free survival. Lanreotide was previously approved for the long-term treatment of acromegalic patients who have had an inadequate response to surgery and/or radiotherapy, or for whom surgery and/or radiotherapy is not an option [74].

It binds to somatostatin receptors (SSTR), specifically SSTR-2 and also to SSTR-5 with a lesser affinity. However, compared to octreotide, this agent is less potent in inhibiting the release of growth hormone from the pituitary gland. Furthermore, lanreotide has an acute effect on decreasing circulating total and free insulin-like growth factor 1 (IGF-I). This agent is usually given as a prolonged-release microparticle or autogel formulation for the treatment of acromegaly and to relieve the symptoms of NETs.

\section{Non-FDA approved somatostatin analogues [75]}

Some of the other clinical trials going on for GEP-NETs are mentioned below:

Sandostatin LAR: It is a somatostatin analogue that adheres to these receptors. This receptor is proposed to be expressed in NETs and is responsible for apoptosis, mediated through a somatostatin receptor. Along with this, it also affects the process of angiogenesis and insulin like growth factor 1 (IGF-1).

\section{Conclusion}

GEP-NETs belong to the class of NETs. It is a rare cancer and MEN 1 is the major predisposing factor associated with them. Alterations and loss of various chromosomes result in the progression of GEP-NETs. Researchers are still challenged in exploring innate and adaptive immune systems. Immunotherapy has shown a promising development in the past few years. Although, there are very few FDA approved immunotherapeutic agents available for GEP-NETs, a number of clinical trials are ongoing for various classes like MABs, adoptive therapy, vaccines and TK inhibitors. The complete perspective of immunotherapy treatment has not been realized and/or utilized. Proper preclinical and clinical designs are the important pillars in understanding the future of immunotherapy in treating cancer patients.

\section{References}

1. Oberg K, Akerström G, Rindi G, Jelic S (2010) Neuroendocrine gastroenteropancreatic tumours: ESMO Clinical Practice Guidelines for diagnosis, treatment and follow-up. Ann Oncol 21: v223-227. [Crossref]

2. Modlin IM, Lye KD, Kidd M (2003) A 5-decade analysis of 13,715 carcinoid tumors. Cancer 97: 934-959. [Crossref]

3. Fraenkel M, Kim MK, FaggianoA, Valk GD (2012) Epidemiology of gastroenteropancreatic neuroendocrine tumours. Best Practice \& Research Clinical Gastroenterology 26: 691-703.

4. Díez M, Teulé A, Salazar R (2013) Gastroenteropancreatic neuroendocrine tumors diagnosis and treatment. Ann Gastroenterol 26: 29-36. [Crossref]

Table 2. Non-FDA approved heat shock protein inhibitors [67]

\begin{tabular}{|c|c|c|c|c|c|}
\hline Cancer Type & $\begin{array}{c}\text { Heat shock protein } \\
\text { inhibitors }\end{array}$ & $\begin{array}{c}\text { Clinical trial identifier } \\
\text { number }\end{array}$ & Phase & Study design \\
\hline NETs & SNX-5422 & NCT02063958 & Phase-I & Safety Study, Open Label \\
\hline
\end{tabular}

Table 3. Non-FDA approved cyokine therapy [68-70]

\begin{tabular}{|c|c|c|c|c|}
\hline & Cytokine Therapy & $\begin{array}{c}\text { Clinical trial identifier } \\
\text { number }\end{array}$ & Phase & Study design \\
\hline Gastrointestinal NETs & Recombinant Interleukin-12 & NCT00004074 & Phase -I & Safety Study, Open Label \\
\hline Gastrointestinal NETs & Recombinant Interferon- alfa & NCT00002470 & Phase-II & Treatment \\
\hline Gastrointestinal NETs & Interferon alfa-2b & NCT00055809 & Phase-II & Efficacy Study, Open Label \\
\hline
\end{tabular}


5. Yao JC, Hassan M, Phan A (2008) One hundred years after "carcinoid": epidemiology of and prognostic factors for neuroendocrine tumors in 35,825 cases in the United States. J ClinOncol 26: 3063-3072.

6. Díez M, Teulé A, Salazar R (2013) Gastroenteropancreatic neuroendocrine tumors: diagnosis and treatment. Ann Gastroenterol 26: 29-36. [Crossref]

7. Lawrence B, Gustafsson BI, Chan A (2011) The epidemiology of gastro entero pancreatic neuroendocrine tumors. Endocrinol Metab Clin North Am 40: 1-18.

8. Chang S, Choi D, Lee SJ (2007) Neuroendocrine neoplasms of the gastrointestinal tract: classification, pathologic basis, and imaging features. Radio Graphics 27: 1667-1679.

9. Hassan MM, Phan A, Li D (2008) Risk factors associated with neuroendocrine tumors: A U.S.- based case-control study. Int J Cancer 123: 867-873.

10. Nascimbeni R, Villanacci V, Di Fabio F (2005) Solitary microcarcinoid of the rectal stump in ulcerative colitis. Neuroendocrinology 81: 400-404.

11. Turaga KK, Kvols LK (2011) Recent progress in the understanding, diagnosis, and treatment of gastroenteropancreatic neuroendocrine tumors. CA Cancer J Clin 61: 113-132.

12. Heller MT, Shah AB (2011) Imaging of neuroendocrine tumors. Radiol Clin North Am 49: 529-548. [Crossref]

13. Toumpanakis CG, Caplin ME (2008) Molecular genetics of gastroenteropancreatic neuroendocrine tumors. Am J Gastroenterol 103: 729-732.

14. Oberg K (2010) Pancreatic endocrine tumors. Semin Oncol 37: 594-618. [Crossref]

15. Kulke MH, Anthony LB, Bushnell DL (2010) NANETS treatment guidelines: well differentiated neuroendocrine tumors of the stomach and pancreas. Pancreas 39: 735-752.

16. Lodish MB, Stratakis CA (2010) Endocrine tumours in neurofibromatosis type 1, tuberous sclerosis and related syndromes. Best Pract Res Clin Endocrinol Metab 24: 439-449.

17. Oberg K, Akerström G, Rindi G (2010) ESMO Guidelines Working Group. Neuroendocrine gastroenteropancreatic tumours: ESMO Clinical Practice Guidelines for diagnosis, treatment and follow-up. Ann Oncol 21: v223-v227.

18. Shehata BM, Stockwell CA, Castellano-Sanchez AA, Setzer S, Schmotzer CL, et al. (2008) Von Hippel-Lindau (VHL) disease: an update on the clinico-pathologic and genetic aspects. Adv Anat Pathol 15: 165-171. [Crossref]

19. Chung CD, Duerr EM (2007) Molecular genetics of neuroendocrine tumors. Best Practice \& Research Clinical Endocrinology \& Metabolism 21: 1-14.

20. Kytola S, Nord B, Elder EE (2002) Alterations of the SDHD gene locus in midgut carcinoids, Merkel cell carcinomas, pheochromocytomas, and abdominal paragangliomas. Genes Chromosomes and Cancer 34: 325-332.

21. Zhao J, de Krijger RR, Meier D (2000) Genomic alterations in well-differentiated gastrointestinal and bronchial neuroendocrine tumors (carcinoids): marked differences indicating diversity in molecular pathogenesis. American Journal of Pathology 157: 1431-1438

22. Tonnies H, Toliat MR, Ramel C (2001) Analysis of sporadic neuroendocrine tumours of the enteropancreatic system by comparative genomic hybridization. Gut 48: 536541.

23. Lollgen RM, Hessman O, Szabo E (2001) Chromosome 18 deletions are common events in classical midgut carcinoid tumors. International Journal of Cancer 92 812-815.

24. Chan AO, Kim SG, Bedeir A, Issa JP, Hamilton SR, et al. (2003) CpG island methylation in carcinoid and pancreatic endocrine tumors. Oncogene 22: 924-934. [Crossref]

25. House MG, Herman JG, Guo MZ (2003) Aberrant hypermethylation of tumor suppressor genes in pancreatic endocrine neoplasms. Annals of Surgery 238: 423431.

26. House MG, Herman JG, Guo MZ (2003) Prognostic value of hMLH1 methylation and microsatellite instability in pancreatic endocrine neoplasms. Surgery 134: 902908.

27. Pfeifer GP, Dammann R (2005) Methylation of the tumor suppressor gene RASSF1A in human tumors. Biochemistry (Mosc) 70: 576-583. [Crossref]

28. Shivakumar L, Minna J, Sakamaki T (2002) The RASSF1A tumor suppressor blocks cell cycle progression and inhibits cyclin D1 accumulation. Molecular and Cellular Biology 22: 4309-4318
29. Pizzi S, Azzoni C, Bottarelli LC (2005) RASSF1A promoter methylation and 3p21.3 loss of heterozygosity are features of foregut, but not midgut and hindgut, malignant endocrine tumours. The Journal of Pathology 206: 409-416.

30. Dammann R, Schagdarsurengin U, Liu L (2003) Frequent RASSF1A promoter hypermethylation and K-ras mutations in pancreatic carcinoma. Oncogene 22: 3806 3812.

31. Stålberg P, Granberg D, Carling T, Wilander E, Eriksson B, et al. (2003) In situ RNA-RNA hybridisation of phospholipase $\mathrm{C}$ beta 3 shows lack of expression in neuroendocrine tumours. Anticancer Res 23: 2227-2232. [Crossref]

32. Sherr CJ (2000) The Pezcoller lecture: cancer cell cycles revisited. Cancer Res 60 3689-3695. [Crossref]

33. Sherr CJ, Roberts JM (1995) Inhibitors of mammalian G cyclin-dependent kinases. Genes Dev 9: 1149-1163. [Crossref]

34. Quelle DE, Zindy F, Ashmun RA, Sherr CJ (1995) Alternative reading frames of the INK4a tumor suppressor gene encode two unrelated proteins capable of inducing cell cycle arrest. Cell 83: 993-1000. [Crossref]

35. Chung DC, Smith AP, Louis DN (1997) Analysis of the retinoblastoma tumour suppressor gene in pancreatic endocrine tumours. Clinical Endocrinology 47: 523528.

36. Kawahara M, Kammori M, Kanauchi H (2002) Immunohistochemical prognostic indicators of gastrointestinal carcinoid tumours. European Journal of Surgical Oncology 28: 140-146

37. Chung DC, Brown SB, Graeme-Cook F (2000) Overexpression of cyclin D1 occurs frequently in human pancreatic endocrine tumors. The Journal of Clinical Endocrinology and Metabolism 85: 4373-4378.

38. Guo SS, Wu X, Shimoide AT (2003) Frequent overexpression of cyclin D1 in sporadic pancreatic endocrine tumours. The Journal of Endocrinology 179: 73-79.

39. FDA label SUTENT ${ }^{\circledR}$ (sunitinib malate). Distributed by Pfizer Labs Division of Pfizer Inc New York, NY 10017.

40. Massachusetts General Hospital. Cabozantinib in advanced pancreatic neuroendocrine and carcinoid tumors. in: clinicaltrials.gov. bethesda (md): national library of medicine (US)

41. National Cancer Institute (NCI). Pazopanib hydrochloride in treating patients with progressive carcinoid tumors. in: clinicaltrials.gov. bethesda (md): national library of medicine (US).

42. Northwestern University: Robert H. lurie cancer center, national comprehensive cancer network, glaxosmithkline. temozolomide and pazopanib hydrochloride in treating patients with advanced pancreatic neuroendocrine tumors that cannot be removed by Surgery. In: ClinicalTrials.gov. Bethesda (MD): National Library of Medicine (US).

43. National Cancer Institute (NCI). sorafenibtosylate in treating patients with progressive metastatic neuroendocrine tumors. in: clinicaltrials.gov. bethesda (md): National Library of Medicine (US).

44. Dana-Farber Cancer Institute: Novartis,Bayer. sorafenib in combination with rad001 in patients with advanced neuroendocrine tumors. In: ClinicalTrials.gov. Bethesda (MD): National Library of Medicine (US).

45. National Cancer Institute (NCI). Trebananib and temsirolimus in treating patients with solid tumors that are metastatic or cannot be removed by surgery. In ClinicalTrials.gov. Bethesda (MD): National Library of Medicine (US).

46. Eastern Cooperative Oncology Group; National Cancer Institute (NCI). AMG 706 and octreotide in treating patients with low-grade neuroendocrine tumors. In: ClinicalTrials.gov. Bethesda (MD): National Library of Medicine (US).

47. Eastern Cooperative Oncology Group; National Cancer Institute (NCI). Vatalanib and octreotide in treating patients with progressive neuroendocrine tumors. In: ClinicalTrials.gov. Bethesda (MD): National Library of Medicine (US).

48. H. Lee Moffitt Cancer Center and Research Institute; national comprehensive cancer network, pfizer.a study of axitinib in advanced carcinoid tumors. In: ClinicalTrials. gov. Bethesda (MD): National Library of Medicine (US).

49. University of Southern California; national cancer institute (nci). regorafenib in treating patients with advanced or metastatic neuroendocrine tumors. In: ClinicalTrials.gov. Bethesda (MD): National Library of Medicine (US).

50. National Cancer Institute (NCI). Gefitinib in treating patients with progressive metastatic neuroendocrine tumors. In: ClinicalTrials.gov. Bethesda (MD): National Library of Medicine (US). 
51. Academic and community cancer research united: national cancer institute (nci). dovitinib lactate in treating patients with pancreatic neuroendocrine tumors. In: ClinicalTrials.gov. Bethesda (MD): National Library of Medicine (US).

52. Jiangsu HengRui Medicine Co., Ltd. cancer institute and hospital, chinese academy of medical sciences. In: ClinicalTrials.gov. Bethesda (MD): National Library of Medicine (US)

53. Washington university school of medicine. VEGFR/PDGFR dual kinase inhibitor $\mathrm{x}-82$ and everolimus for treating patients with pancreatic neuroendocrine tumors. In: ClinicalTrials.gov. Bethesda (MD): National Library of Medicine (US).

54. Novartis pharmaceuticals. efficacy and safety of bez235 compared to everolimus in patients with advanced pancreatic neuroendocrine tumors (MACS1938). In: ClinicalTrials.gov. Bethesda (MD): National Library of Medicine (US).

55. Novartis pharmaceuticals. study of safety and efficacy of byl719 with everolimus or byl719 with everolimus and exemestane in advanced breast cancer patients, renal cell cancer and pancreatic tumors. In: ClinicalTrials.gov. Bethesda (MD): National Library of Medicine (US).

56. FDA label AFINITOR ${ }^{\circledR}$ (everolimus). manufactured by: novartis pharma stein agstein, switzerland. distributed by: novartis pharmaceuticals corporation east hanover, New Jersey 07936

57. National Cancer Institute (NCI). temsirolimus and bevacizumab in treating patients with advanced endometrial, ovarian, liver, carcinoid, or islet cell cancer. In: ClinicalTrials.gov. Bethesda (MD): National Library of Medicine (US).

58. SCRI development innovations, llc; onyx pharmaceuticals. In: ClinicalTrials.gov. Bethesda (MD): National Library of Medicine (US).

59. Ohio state university comprehensive cancer center; national cancer institute (nci). ps-341 in treating patients with metastatic neuroendocrine tumors. In: ClinicalTrials. gov. Bethesda (MD): National Library of Medicine (US).

60. National Cancer Institute (NCI). Bevacizumab and PEG-Interferon Alfa-2b in treating patients with metastatic or unresectable carcinoid tumors. In: ClinicalTrials. gov. Bethesda (MD): National Library of Medicine (US).

61. National Cancer Institute (NCI). Everolimus and octreotide acetate with or without bevacizumab in treating patients with locally advanced or metastatic pancreatic neuroendocrine tumors that cannot be removed by surgery. In: ClinicalTrials.gov. Bethesda (MD): National Library of Medicine (US).

62. Dana-Farber cancer institute; brigham and women's hospital, massachusetts general hospital, h. lee moffitt cancer center and research institute, amgen. amg 479 in advanced carcinoid and pancreatic neuroendocrine tumors. In: ClinicalTrials.gov. Bethesda (MD): National Library of Medicine (US).
63. Dana-Farber cancer institute; brigham and women's hospital, massachusetts general hospital, h. lee moffitt cancer center and research institute, amgen amg 479 in advanced carcinoid and pancreatic neuroendocrine tumors.In: ClinicalTrials.gov. Bethesda (MD): National Library of Medicine (US).

64. Genentech, Inc. Phase II study of pertuzumab and erlotinib for metastatic or unresectable neuroendocrine tumors. In: ClinicalTrials.gov. Bethesda (MD): National Library of Medicine (US).

65. National Cancer Institute (NCI); National Cancer Institute (NCI). Cixutumumab, Everolimus, and Octreotide Acetate in Treating Patients with Advanced Low to Intermediate Grade Neuroendocrine Carcinoma. In: ClinicalTrials.gov. Bethesda (MD): National Library of Medicine (US).

66. Basu B, Sirohi B, Corrie P (2010) Systemic therapy for neuroendocrine tumours of gastroenteropancreatic origin. Endocrine-Related Cancer 17: R75-R90.

67. Esanex Inc, Esanex Inc.Safety and Pharmacology of SNX-5422 plus everolimus in subjects with neuroendocrine tumors. In: ClinicalTrials.gov. Bethesda (MD): National Library of Medicine (US).

68. National Cancer Institute (NCI). interleukin-12 and trastuzumab in treating patient with cancer that has high levels of HER2/Neu. In: ClinicalTrials.gov. Bethesda (MD): National Library of Medicine (US).

69. Mid-Atlantic oncology program; cancer biotherapy research group. fluorouracil plus interferon alfa in treating patients with advanced metastatic carcinoid tumors. In ClinicalTrials.gov. Bethesda (MD): National Library of Medicine (US).

70. National Cancer Institute (NCI). bevacizumab and peg-interferon alfa-2b in treating patients with metastatic or unresectable carcinoid tumors. In: ClinicalTrials.gov. Bethesda (MD): National Library of Medicine (US).

71. National Cancer Institute (NCI). romidepsin in treating patients with lymphoma, chronic lymphocytic leukemia, or solid tumors with liver dysfunction. In: ClinicalTrials.gov. Bethesda (MD): National Library of Medicine (US).

72. Dana-Farber Cancer Institute. ziv-aflibercept for advanced progressive carcinoid tumors. In: ClinicalTrials.gov. Bethesda (MD): National Library of Medicine (US).

73. National Cancer Institute (NCI). Ziv-Aflibercept in treating and computed tomography perfusion imaging in predicting response in patients with pancreatic neuroendocrine tumors that are metastatic or cannot be removed by surgery. In: ClinicalTrials.gov

74. US food and drug administration (FDA) http://www.fda.gov/Drugs/ InformationOnDrugs/ApprovedDrugs/ucm427065.htm

75. Fondazione IRCCS istituto nazionale dei tumori, milano; fondazione irccs istituto nazionale dei tumori, milano. activity and safety of everolimus+octreotide lar+metformin in advanced pancreatic well-differentiated NETs (MetNET1). In: ClinicalTrials.gov. Bethesda (MD): National Library of Medicine (US).

Copyright: (C2019 Allen T. This is an open-access article distributed under the terms of the Creative Commons Attribution License, which permits unrestricted use, distribution, and reproduction in any medium, provided the original author and source are credited. 